\title{
Eine Klinik stellt sich vor: Klinik für Dermatologie und Venerologie der Georg-August-Universität Göttingen
}

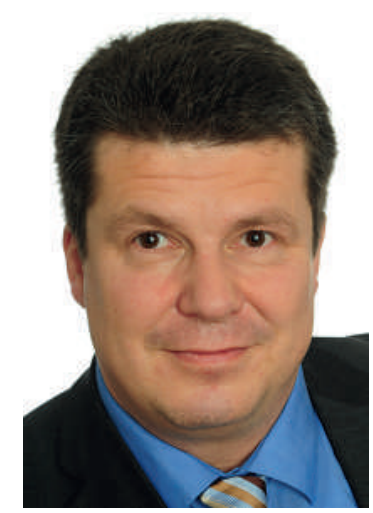

Prof. Dr. med. Michael P. Schön
Die Göttinger Universitäts-Hautklinik blickt auf eine mehr als 90-jährige Geschichte zurück, die von verschiedenen klinischen und wissenschaftlichen Schwerpunkten geprägt war. Heute ist die Klinik mit einem gut eingespielten Team motivierter und hoch qualifizierter Mitarbeiter, die das gesamte Spektrum unseres Faches sowie mehrere Zusatz-Qualifikationen abdecken, modern und leistungsstark. Die Klinik verfügt, neben der Weiterbildungsermächtigung für Dermatologie und Venerologie, auch über diejenigen für Allergologie, Phlebologie, Dermatohistologie sowie medikamentöse Tumortherapie.

Die Onkologie und die Dermatochirurgie sind wichtige Bausteine der klinischen Versorgung. Die gesamte dermatologische Chirurgie - auch große Operationen, Sentinel-Lymphknoten-Biopsien sowie ausgedehnte Lymphadenektomien werden unter oberärztlicher Leitung durch Prof. Dr. L. Kretschmer durchgeführt. Enge Zusammenarbeit mit niedergelassenen Kollegen, interdisziplinäre Kooperation sowie die Einbindung in das Göttinger „Comprehensive Cancer Center“ (G-CCC) sind dabei selbstverständlich.

Ein weiterer Schwerpunkt ist die Phlebologie. Unter oberärztlicher Leitung durch Dr. M. Zutt und Prof. Dr. L. Kretschmer wird das gesamte Spektrum der konservativen und operativen Phlebologie sowie der modernen Wundversorgung angeboten.

Die histologische Begutachtung klinikeigener und eingesandter Präparate wird auf höchstem Niveau durchgeführt. Diese Histologie-Abteilung expandierte im vergangenen Jahr und wir sind sehr daran interessiert, weitere Kooperationen mit niedergelassenen Kollegen als Einsender histologischer Präparate zu etablieren. Unter oberärztlicher Leitung durch Dr. H.-P. Bertsch wird nicht nur eine zuverlässige und rasche Bearbeitung der „Standard“-Histologien gewährleistet, sondern auch die komplette Lymphom- und SentinelLymphknoten-Diagnostik durchgeführt. Damit wird das gesamte Spektrum der dermatologischen Onkologie - operativ, konservativ und histopathologisch - angeboten.

Die allergologische Abteilung betreut jährlich etwa 3500 Patienten unter Vorhaltung sämtlicher diagnostischer und therapeutischer Verfahren. Die überregionale Bedeutung der Göttinger Allergologie zeigt sich auch an der hohen Teilnehmerzahl bei der jährlich von Prof. Dr. T. Fuchs organisierten Allergologischen Wintertagung.

Eine weitere Besonderheit der Göttinger dermatologischen Landschaft ist der Informationsverbund Dermatologischer Kliniken (IVDK), der unter Leitung durch Prof. Dr. A. Schnuch seinen Sitz als
An-Institut an der Hautklinik hat. Der IVDK, der inzwischen Daten von mehr als 165000 Patienten gespeichert hat, hat national und international einen unschätzbaren Beitrag zur epidemiologischen Forschung bei Kontaktallergien geleistet. Patienten mit oftmals komplexen Autoimmunerkrankungen werden unter oberärztlicher Leitung durch Dr. C. Seitz erstklassig versorgt. Von der molekularen und immunologischen Diagnostik bis zu innovativen Therapien ist hier - in enger inerdisziplinärer Kooperation - ein weiterer klinischer Schwerpunkt etabliert.

Neben der großen Poliklinik, die oberärztlich von Prof. Dr. Steffen Emmert geleitet wird, werden verschiedene Spezialsprechstunden und klinische Studien für Patienten mit Psoriasis, Kollagenosen, blasenbildenden Autoimmunerkrankungen, Neurodermitis oder Tumoren, regelmäßig angeboten.

Wissenschaftliche Schwerpunkte liegen im onkologischen sowie entzündungsbiologisch-immunologischen Bereich: Extern geförderte Projekte umfassen Untersuchungen zu DNA-Reparaturmechanismen bei der Tumorentstehung, zellulären Signalwegen bei Chemoresistenz maligner Tumoren, antiangiogener Gentherapie bei entzündlichen Erkrankungen, Steuerung der Antigenpräsentation bei der Tumortherapie, Rekrutierung von Entzündungszellen sowie Polymorphismen bei entzündlichen und malignen Erkrankungen. Mit dem Bezug neuer Laborräume, der Anschaffung neuer wissenschaftlicher Geräte, sowie durch intensive Kooperationen steht in Göttingen eine exzellente Infrastruktur zur erfolgreichen Bearbeitung der Projekte zur Verfügung. Die Göttinger Universitäts-Hautklinik betreibt eine moderne, wissenschaftlich fundierte Dermatologie in Kooperation und offenem Kontakt mit niedergelassenen Kollegen und anderen Fachdisziplinen. Natürlich bleibt auch unsere Klinik nicht unberührt von ökonomischen Herausforderungen unseres Gesundheitssystems; wir haben diese angenommen, um unsere Patientenversorgung zu optimieren und weiter zu verbessern. Durch gemeinsame Anstrengung sämtlicher Mitarbeiter wurde so im vergangenen Jahr eine deutliche Leistungssteigerung erreicht, die letztlich auch der Entwicklung unserer Klinik nützen wird.

Ich möchte die Leser nun einladen, unsere schöne Klinik näher kennenzulernen und wünsche Ihnen bei der Lektüre des Themenheftes viel Freude neue Kontakte und Kooperationen, die sich hierdurch ergeben, sind uns sehr willkommen.

Mit den besten kollegialen Grüßen Michael P. Schön 